PROCEEDINGS OF THE

AMERICAN MATHEMATICAL SOCIETY

Volume 131, Number 1, Pages 155-164

S 0002-9939(02)06491-2

Article electronically published on May 13, 2002

\title{
APPLICATION OF A RIESZ-TYPE FORMULA TO WEIGHTED BERGMAN SPACES
}

\author{
ALI ABKAR
}

(Communicated by Juha M. Heinonen)

\begin{abstract}
Let $\mathbb{D}$ denote the unit disk in the complex plane. We consider a class of superbiharmonic weight functions $w: \mathbb{D} \rightarrow \mathbb{R}^{+}$whose growth are subject to the condition $0 \leq w(z) \leq C(1-|z|)$ for some constant $C$. We first establish a Reisz-type representation formula for $w$, and then use this formula to prove that the polynomials are dense in the weighted Bergman space with weight $w$.
\end{abstract}

\section{INTRODUCTION}

We denote by $\mathbb{D}$ the unit disk and by $\mathbb{T}$ the unit circle in the complex plane. A weight function, or simply a weight, in $\mathbb{D}$ is any continuous positive function $w: \mathbb{D} \rightarrow[0,+\infty[$.

The problem. A real-valued function $w$ defined on the unit disk $\mathbb{D}$ is said to be superbiharmonic provided that $w$ is locally integrable and the bi-laplacian $\Delta^{2} w$ is a positive distribution on $\mathbb{D}$; here $\Delta$ stands for the Laplace operator defined by

$$
\Delta=\Delta_{z}=\frac{\partial^{2}}{\partial z \partial \bar{z}}=\frac{1}{4}\left(\frac{\partial^{2}}{\partial x^{2}}+\frac{\partial^{2}}{\partial y^{2}}\right), \quad z=x+i y .
$$

We consider a class of superbiharmonic weight functions $w$ satisfying condition

$$
0 \leq w(z) \leq C(1-|z|), \quad \text { for every } \quad z \in \mathbb{D},
$$

for some constant $C$. We shall prove that if $w$ is a superbiharmonic function satisfying condition (A), then $w$ enjoys a Riesz-type representation formula in terms of the biharmonic Green function for the operator $\Delta^{2}$, and the harmonic compensator. In the following we shall briefly introduce these notions.

The biharmonic Green function for the operator $\Delta^{2}$ in the unit disk is the function $\Gamma(z, \zeta)$ which solves, for fixed $\zeta$, the following boundary value problem:

$$
\begin{cases}\Delta_{z}^{2} \Gamma(z, \zeta)=\delta_{\zeta}(z) & z \in \mathbb{D} \\ \Gamma(z, \zeta)=0, & z \in \mathbb{T} \\ \partial_{n(z)} \Gamma(z, \zeta)=0, & z \in \mathbb{T}\end{cases}
$$

Received by the editors August 16, 2001.

2000 Mathematics Subject Classification. Primary 31A30; Secondary 30E10, 30H05, 46E10.

This research was supported in part by a grant from the Institute for Theoretical Physics and Mathematics (IPM), Tehran, Iran. 
where the symbol $\delta_{\zeta}$ stands for the unit point mass at $\zeta \in \mathbb{D}$, and $\partial_{n(z)}$ denotes the inward normal derivative in the sense of distributions. It is well-known [4] that the biharmonic Green function has the form

$$
\Gamma(z, \zeta)=|z-\zeta|^{2} \log \left|\frac{z-\zeta}{1-\bar{\zeta} z}\right|^{2}+\left(1-|z|^{2}\right)\left(1-|\zeta|^{2}\right), \quad(z, \zeta) \in \mathbb{D} \times \mathbb{D} .
$$

We define the harmonic compensator $H(\zeta, z)$ by

$$
H(\zeta, z)=\left(1-|z|^{2}\right) P(z, \zeta)=\frac{\left(1-|z|^{2}\right)^{2}}{|1-z \bar{\zeta}|^{2}}, \quad(\zeta, z) \in \mathbb{T} \times \mathbb{D},
$$

where $P(z, \zeta)$ denotes the Poisson kernel for the unit disk. It turns out that superbiharmonic functions $w$ satisfying condition (A) can be represented by a Riesz-type formula which takes into account the growth of $w$ as $z$ approaches the boundary. Indeed, we shall see that

$$
w(z)=\int_{\mathbb{D}} \Gamma(z, \zeta) \Delta^{2} w(\zeta) d A(\zeta)+\int_{\mathbb{T}} H(\zeta, z) \partial_{n(\zeta)} w(\zeta) d \sigma(\zeta), \quad z \in \mathbb{D}
$$

where $d A=\pi^{-1} d x d y$ is the normalized area measure in $\mathbb{D}$ and $d \sigma=(2 \pi)^{-1} d \theta$ denotes the normalized arc-length measure on $\mathbb{T}$. Moreover, the normal derivative in the second term should be understood in a generalized sense: $\partial_{n(\zeta)} w(\zeta) d \sigma(\zeta)$ is the weak-star limit of a family of positive continuous functions on the unit circle, so that it can be identified with a positive measure on the unit circle $\mathbb{T}$.

Application to weighted Bergman spaces. We say that a function $f$, analytic in $\mathbb{D}$, belongs to the weighted Bergman space $L_{a}^{p}(\mathbb{D}, w), 0<p<+\infty$, provided that the following integral is finite:

$$
\|f\|_{L_{a}^{p}(\mathbb{D}, w)}^{p}=\int_{\mathbb{D}}|f(z)|^{p} w(z) d A(z)<+\infty .
$$

If $1 \leq p<+\infty$, it follows that $L_{a}^{p}(\mathbb{D}, w)$ is a Banach space of analytic functions with norm $\|\cdot\|_{L_{a}^{p}(\mathbb{D}, w)}$, and for $0<p<1$, it is a quasi-Banach space. For $p=2$, the space $L_{a}^{2}(\mathbb{D}, w)$ is a Hilbert space with the inner product

$$
\langle f, g\rangle_{L_{a}^{2}(\mathbb{D}, w)}=\int_{\mathbb{D}} f(z) \overline{g(z)} w(z) d A(z), \quad f, g \in L_{a}^{2}(\mathbb{D}, w) .
$$

In the case where the weight function $w$ is identically 1 , the corresponding space is called the Bergman space and is denoted by $L_{a}^{p}(\mathbb{D})$. Note that condition $(\mathrm{A})$ entails

$$
\int_{\mathbb{D}} w(z) d A(z)<+\infty
$$

which guarantees that the polynomials are contained in the weighted Bergman space $L_{a}^{p}(\mathbb{D}, w)$.

We devote the last section to an application of the representation formula (1-1) to the weighted Bergman space $L_{a}^{p}(\mathbb{D}, w)$ whose (non-radial) weight $w$ is superbiharmonic and satisfies condition (A). It is shown that the polynomials are dense in such weighted Bergman spaces. We remark that if the weight function $w$ is radial ( $w$ depends only on $|z|$ ), the result is well-known [9]. The question of weighted polynomial approximation for such weights was raised by H. Hedenmalm in [5], p. 114. We answer this question in the positive. 
If we weaken condition (A) above, it is possible to find a representation formula for $w$ with one more term. This is done in [1], where the author joint with Hedenmalm succeeded to establish a Riesz representation formula for $w$.

\section{A RIESZ-TyPE REPRESENTATION FORMUla}

In this section we will find a representation formula for a superbiharmonic function $w$ satisfying the condition $0 \leq w(z) \leq C(1-|z|)$ for some constant $C$. The following lemma asserts that $\Delta^{2} w(z)$ is integrable against the area measure $\left(1-|z|^{2}\right)^{2} d A(z)$ in the unit disk. The (rather long) proof uses the assumption (A) on $w$, twice the application of Green's formula together with some sharp estimates of the laplacians and the normal derivatives involved. We omit the details and refer the reader to [1], Lemma 3.1.

Lemma 2.1. Let $w$ be a superbiharmonic function satisfying $0 \leq w(z) \leq C(1-|z|)$. Then

$$
\int_{\mathbb{D}}\left(1-|z|^{2}\right)^{2} \Delta^{2} w(z) d A(z)<+\infty .
$$

Proof. The assertion is a consequence of Lemma $3.1 \mathrm{in} \mathrm{[1].}$

In the following lemma we collect some facts on the biharmonic Green function for the unit disk. The proof of parts (c) and (d) can be found in [2], Lemma 2.3. Part (a) can be verified by a direct argument using the defining formula for $\Gamma(z, \zeta)$, and finally, (b) is an immediate consequence of (a).

Lemma 2.2. Let $\Gamma(z, \zeta)$ denote the biharmonic Green function for the unit disk. Then

(a) $\Gamma(z, \zeta)$ has the power series representation

$$
\Gamma(z, \zeta)=\sum_{n=0}^{\infty} \frac{\left[\left(1-|z|^{2}\right)\left(1-|\zeta|^{2}\right)\right]^{n+2}}{(n+1)(n+2)|1-\bar{z} \zeta|^{2(n+1)}}, \quad(z, \zeta) \in \mathbb{D} \times \mathbb{D},
$$

(b) $\Gamma(z, \zeta)>0$, for every $(z, \zeta) \in \mathbb{D} \times \mathbb{D}$,

(c) for every $(z, \zeta) \in \mathbb{D} \times \mathbb{D}$ we have

$$
\frac{1}{2} \frac{\left(1-|z|^{2}\right)^{2}\left(1-|\zeta|^{2}\right)^{2}}{|1-\bar{\zeta} z|^{2}} \leq \Gamma(z, \zeta) \leq \frac{\left(1-|z|^{2}\right)^{2}\left(1-|\zeta|^{2}\right)^{2}}{|1-\bar{\zeta} z|^{2}}
$$

(d) for every $(z, \zeta) \in \mathbb{D} \times \mathbb{D}$ we have

$$
(1-|z|)^{2}\left(1-|\zeta|^{2}\right)^{2} \leq 2 \Gamma(z, \zeta) \leq(1+|z|)^{2}\left(1-|\zeta|^{2}\right)^{2} .
$$

We now combine the preceding lemmas and obtain the following proposition.

Proposition 2.3. Let $w$ be a superbiharmonic weight function satisfying the condition $0 \leq w(z) \leq C(1-|z|)$. Let $\Gamma(z, \zeta)$ denote the biharmonic Green function for the unit disk. Then

$$
\int_{\mathbb{D}} \Gamma(z, \zeta) \Delta^{2} w(\zeta) d A(\zeta)<+\infty, \quad z \in \mathbb{D}
$$

Proof. According to Lemma 2.2(d), for every $(z, \zeta) \in \mathbb{D} \times \mathbb{D}$,

$$
(1-|z|)^{2}\left(1-|\zeta|^{2}\right)^{2} \leq 2 \Gamma(z, \zeta) \leq(1+|z|)^{2}\left(1-|\zeta|^{2}\right)^{2} .
$$


Hence

$$
\Gamma(z, \zeta) \leq 2\left(1-|\zeta|^{2}\right)^{2}, \quad(z, \zeta) \in \mathbb{D} \times \mathbb{D}
$$

from which follows

$$
\int_{\mathbb{D}} \Gamma(z, \zeta) \Delta^{2} w(\zeta) d A(\zeta) \leq 2 \int_{\mathbb{D}}\left(1-|\zeta|^{2}\right)^{2} \Delta^{2} w(\zeta) d A(\zeta)<+\infty
$$

in accordance with Lemma 2.1.

Proposition 2.4. Let $w$ be a superbiharmonic weight function satisfying the condition $0 \leq w(z) \leq C(1-|z|)$. Define the function $v$ by the formula:

$$
v(z)=\int_{\mathbb{D}} \Gamma(z, \zeta) \Delta^{2} w(\zeta) d A(\zeta), \quad z \in \mathbb{D} .
$$

Then for every integer $k$ we have

$$
\lim _{r \rightarrow 1^{-}} \int_{\mathbb{T}} \frac{\bar{z}^{k} v(r z)}{1-r^{2}} d \sigma(z)=0
$$

In fact,

$$
\sup _{k}\left|\int_{\mathbb{T}} \frac{\bar{z}^{k} v(r z)}{1-r^{2}} d \sigma(z)\right| \leq \int_{\mathbb{T}} \frac{v(r z)}{1-r^{2}} d \sigma(z) \rightarrow 0, \quad \text { as } \quad r \rightarrow 1^{-} .
$$

Proof. For an integer $k$ and $0<r<1$ we define

$$
C(k, r)=\int_{\mathbb{T}} \frac{\bar{z}^{k} v(r z)}{1-r^{2}} d \sigma(z) .
$$

We first make the following simple, but important, observation. Since the function $v(r z) /\left(1-r^{2}\right)$ is nonnegative, it follows that for every integer $k$ we have

$$
0 \leq|C(k, r)| \leq \int_{\mathbb{T}}\left|\frac{\bar{z}^{k} v(r z)}{1-r^{2}}\right| d \sigma(z)=C(0, r) .
$$

Hence it suffices to verify the validity of the statement of the proposition for $k=0$; that is to show that $C(0, r) \rightarrow 0$ as $r \rightarrow 1^{-}$. To this end, we note that

$$
0 \leq C(0, r)=\int_{\mathbb{T}} \int_{\mathbb{D}} \frac{\Gamma(r z, \zeta)}{1-r^{2}} \Delta^{2} w(\zeta) d A(\zeta) d \sigma(z) .
$$

It now follows from Fubini's theorem and Lemma 2.2(c) that

$$
\begin{aligned}
0 \leq C(0, r) & \leq \int_{\mathbb{D}} \int_{\mathbb{T}} \frac{\left(1-r^{2}|z|^{2}\right)^{2}\left(1-|\zeta|^{2}\right)^{2}}{\left(1-r^{2}\right)|1-r z \bar{\zeta}|^{2}} \Delta^{2} w(\zeta) d \sigma(z) d A(\zeta) \\
& =\left(\int_{\mathbb{D}}\left(1-|\zeta|^{2}\right)^{2} \Delta^{2} w(\zeta) d A(\zeta)\right) \int_{\mathbb{T}} \frac{1-r^{2}}{|1-r z \bar{\zeta}|^{2}} d \sigma(z)
\end{aligned}
$$

But

$$
\int_{\mathbb{T}} \frac{1}{|1-r z \bar{\zeta}|^{2}} d \sigma(z)=\frac{1}{1-|r \zeta|^{2}} \int_{\mathbb{T}} \frac{1-|r \zeta|^{2}}{|z-r \zeta|^{2}} d \sigma(z)=\frac{1}{1-|r \zeta|^{2}}
$$

This together with $(2-2)$ yields

$$
0 \leq C(0, r) \leq \int_{\mathbb{D}} \frac{1-r^{2}}{1-r^{2}|\zeta|^{2}}\left(1-|\zeta|^{2}\right)^{2} \Delta^{2} w(\zeta) d A(\zeta)
$$


To deal with the area integral appearing in $(2-3)$ we write

$$
d \lambda(\zeta)=\left(1-|\zeta|^{2}\right)^{2} \Delta^{2} w(\zeta) d A(\zeta), \quad \zeta \in \mathbb{D}
$$

We note that $d \lambda(\zeta)$ is a positive measure on the unit disk, and according to Lemma 2.1 the integral of the constant function 1 against the measure $d \lambda$ is finite. Hence $(2-3)$ can be written as

$$
0 \leq C(0, r) \leq \int_{\mathbb{D}} \frac{1-r^{2}}{1-r^{2}|\zeta|^{2}} d \lambda(\zeta)
$$

Since the integrand in $(2-4)$ is nonnegative and bounded from above by 1 , we can apply the dominated convergence theorem to the right-hand side of $(2-4)$ to obtain

$$
0 \leq \lim _{r \rightarrow 1^{-}} C(0, r) \leq \lim _{r \rightarrow 1^{-}} \int_{\mathbb{D}} \frac{1-r^{2}}{1-r^{2}|\zeta|^{2}} d \lambda(\zeta)=0,
$$

from which the proposition follows.

Corollary 2.5. With the same conditions as in the preceding proposition

$$
\sup _{k}\left|\int_{\mathbb{T}} \bar{z}^{k} v(r z) d \sigma(z)\right|=o(1-r) \quad \text { as } \quad r \rightarrow 1^{-} \text {. }
$$

We have prepared the ground for the main result of this section.

Theorem 2.6. Let $w$ be a superbiharmonic weight function satisfying the condition $0 \leq w(z) \leq C(1-|z|)$. Then

$$
w(z)=\int_{\mathbb{D}} \Gamma(z, \zeta) \Delta^{2} w(\zeta) d A(\zeta)+\int_{\mathbb{T}} H(\zeta, z) \partial_{n(\zeta)} w(\zeta) d \sigma(\zeta), \quad z \in \mathbb{D},
$$

where $H(\zeta, z)$ is the harmonic compensator and $\partial_{n} w$ is the generalized normal derivative of $w$; in the sense that $\partial_{n(\zeta)} w(\zeta) d \sigma(\zeta)$ is a positive measure on the unit circle.

Proof. We define the real-valued function $v$ by

$$
v(z)=\int_{\mathbb{D}} \Gamma(z, \zeta) \Delta^{2} w(\zeta) d A(\zeta), \quad z \in \mathbb{D} .
$$

According to Proposition 2.3 the function $v$ is well-defined. Moreover, $v$ is nonnegative, because $\Gamma(z, \zeta)$ is positive in $\mathbb{D} \times \mathbb{D}$, and $\Delta^{2} w \geq 0$ by our assumption.

We shall first verify that the weight function $w$ can be written as

$$
w(z)=v(z)+\left(1-|z|^{2}\right) h(z), \quad z \in \mathbb{D},
$$

where $h$ is a harmonic function in the unit disk. It is clear that $w-v$ is a biharmonic function, hence according to the Almansi representation formula for biharmornic functions (see 6], Lemma 3.1) there exist two real-valued harmonic functions $h$ and $u$ such that

$$
w(z)-v(z)=u(z)+\left(1-|z|^{2}\right) h(z), \quad z \in \mathbb{D}
$$

We fix a real number $r, 0<r<1$, and an integer $n$. Then we have

$$
w(r z)-v(r z)=u(r z)+\left(1-r^{2}\right) h(r z), \quad z \in \mathbb{T}
$$


from which it follows that

$$
\begin{aligned}
& \int_{\mathbb{T}} \bar{z}^{n}(w(r z)-v(r z)) d \sigma(z) \\
&=\int_{\mathbb{T}} \bar{z}^{n} u(r z) d \sigma(z)+\left(1-r^{2}\right) \int_{\mathbb{T}} \bar{z}^{n} h(r z) d \sigma(z) \\
&=\widehat{u}_{r}(n)+\left(1-r^{2}\right) \widehat{h}_{r}(n) .
\end{aligned}
$$

Here $\hat{u}(n)$ stands for the $n$-th Fourier coefficient of $u$; moreover, $u_{r}(z)=u(r z)$. Since $u$ and $h$ are harmonic, it follows that

$$
\int_{\mathbb{T}} \bar{z}^{n} w(r z) d \sigma(z)-\int_{\mathbb{T}} \bar{z}^{n} v(r z) d \sigma(z)=r^{|n|} \widehat{u}(n)+\left(1-r^{2}\right) r^{|n|} \widehat{h}(n) .
$$

Letting now $r \rightarrow 1^{-}$, we see that the right-hand side of $(2-5)$ tends to $\widehat{u}(n)$. As for the left-hand side of (2-5) we see that the first integral tends to zero, as $r \rightarrow 1^{-}$. Indeed, we know from our assumption on $w$ that

$$
0 \leq w(r z) \leq C(1-r|z|)=C(1-r), \quad \text { for } \quad z \in \mathbb{T},
$$

and hence

$$
0 \leq\left|\int_{\mathbb{T}} \bar{z}^{n} w(r z) d \sigma(z)\right| \leq \int_{\mathbb{T}} w(r z) d \sigma(z) \leq C(1-r) \rightarrow 0, \quad \text { as } \quad r \rightarrow 1^{-} .
$$

The fact that the second integral on the left-hand side of $(2-5)$ approaches zero as $r \rightarrow 1^{-}$follows from Corollary 2.5. Hence for any integer $n$, we have $\widehat{u}(n)=0$, from which it follows that $u$ is identically zero. This implies that

$$
w(z)-v(z)=\left(1-|z|^{2}\right) h(z), \quad z \in \mathbb{D},
$$

where $h$ is some harmonic function in the unit disk $\mathbb{D}$.

We fix once again $0<r<1$ and use $(2-6)$ to write

$$
\frac{w(r z)}{1-r^{2}}-\frac{v(r z)}{1-r^{2}}=h(r z), \quad z \in \mathbb{T} .
$$

It then follows from Proposition 2.4 that for every integer $k$ we have

$$
\lim _{r \rightarrow 1^{-}} \int_{\mathbb{T}} \bar{z}^{k} \frac{w(r z)}{1-r^{2}} d \sigma(z)=\lim _{r \rightarrow 1^{-}} \int_{\mathbb{T}} \bar{z}^{k} h(r z) d \sigma(z)=\widehat{h}(k) .
$$

Note that the limit on the right-hand side of (2-8) exists, since $h$ is a harmonic function. In fact the equality (2-8) holds for every trigonometric polynomial $p$ on the unit circle, that is,

$$
\lim _{r \rightarrow 1^{-}} \int_{\mathbb{T}} p(z) \frac{w(r z)}{1-r^{2}} d \sigma(z)=\lim _{r \rightarrow 1^{-}} \int_{\mathbb{T}} p(z) h(r z) d \sigma(z) .
$$

We shall see that

$$
\sup _{0<r<1}\left\|h_{r}\right\|_{L^{1}(\mathbb{T})}<+\infty .
$$

To see this, we first use $(2-7)$ to write

$$
\int_{\mathbb{T}}|h(r z)| d \sigma(z) \leq \int_{\mathbb{T}} \frac{w(r z)}{1-r^{2}} d \sigma(z)+\int_{\mathbb{T}} \frac{v(r z)}{1-r^{2}} d \sigma(z) .
$$

The first integral in $(2-11)$ is bounded by the constant 1 , as was observed earlier. The uniform boundedness of the second integral in (2-11) follows from Proposition 2.4 for $k=0$. Hence $(2-10)$ holds. This $L^{1}$-boundedness of the functions $h_{r}$ implies 
that there exists a unique real-valued Borel measure $\mu$ on the unit circle such that $h$ is the Poisson integral of this measure (see [10], Theorem 11.30); or

$$
h(z)=\int_{\mathbb{T}} \frac{1-|z|^{2}}{|1-\bar{\zeta} z|^{2}} d \mu(\zeta), \quad z \in \mathbb{D} .
$$

Moreover, the measure $\mu$ is the weak-star limit of the measures $d \mu_{r}=h_{r} d \sigma$ (see [8], p. 33). This means that for every trigonometric polynomial $p$ on the unit circle we have

$$
\begin{aligned}
\int_{\mathbb{T}} p(z) d \mu(z)=\lim _{r \rightarrow 1^{-}} & \int_{\mathbb{T}} p(z) h_{r}(z) d \sigma(z) \\
& =\lim _{r \rightarrow 1^{-}} \int_{\mathbb{T}} p(z) \frac{w(r z)}{1-r^{2}} d \sigma(z)-\lim _{r \rightarrow 1^{-}} \int_{\mathbb{T}} p(z) \frac{v(r z)}{1-r^{2}} d \sigma(z) .
\end{aligned}
$$

It is implicit in (2-9) that the last limit in the above displayed formula is zero from which it follows that

$$
\int_{\mathbb{T}} p(z) d \mu(z)=\lim _{r \rightarrow 1^{-}} \int_{\mathbb{T}} p(z) \frac{w(r z)}{1-r^{2}} d \sigma(z) .
$$

Now, (2-13) implies that for every nonnegative trigonometric polynomial $p$ on the unit circle

$$
\int_{\mathbb{T}} p(z) d \mu(z) \geq 0
$$

proving that $\mu$ is a positive measure on $\mathbb{T}$. This together with (2-12) implies that $h$ is a positive harmonic function in the unit disk. Therefore we obtain from $(2-6)$

$$
0 \leq w(z)-v(z)=\left(1-|z|^{2}\right) h(z) \leq w(z) \leq C(1-|z|), \quad z \in \mathbb{D},
$$

so that $0 \leq h(z) \leq C$, that is, $h$ is a nonnegative bounded harmonic function in the unit disk. It follows from $(2-6)$ and $(2-12)$ that for every $z \in \mathbb{D}$,

$$
\begin{aligned}
w(z)-v(z)=\left(1-|z|^{2}\right) h(z) & =\int_{\mathbb{T}} \frac{\left(1-|z|^{2}\right)^{2}}{|1-\bar{\zeta} z|^{2}} d \mu(\zeta) \\
& =\int_{\mathbb{T}} H(\zeta, z) d \mu(\zeta),
\end{aligned}
$$

where $H(\zeta, z)$ is the harmonic compensator defined by

$$
H(\zeta, z)=\left(1-|z|^{2}\right) P(z, \zeta)=\frac{\left(1-|z|^{2}\right)^{2}}{|1-\bar{\zeta} z|^{2}}, \quad(\zeta, z) \in \mathbb{T} \times \mathbb{D}
$$

in which $P(z, \zeta)$ denotes the Poisson kernel for the unit disk.

The measure $\mu$ in the representation formula (2-14) was obtained as a weakstar limit of the functions $w_{r}(z) /\left(1-r^{2}\right)$, for $z \in \mathbb{T}$. This weak-star limit can be regarded as the generalized normal derivative of the function $w$ on the boundary. The proof of the theorem is now complete.

\section{Applichtion to weighted Bergman spaces}

We start with a lemma which says that the harmonic compensator has some kind of monotonicity property with respect to its second argument. The proof uses the method of [2], p. 285 (see also [7], p. 64, where the result is attributed to the current author). 
Lemma 3.1. Let $z=e^{i \theta} \in \mathbb{T}$ be fixed, and consider

$$
H\left(e^{i \theta}, \zeta\right)=\frac{\left(1-|\zeta|^{2}\right)^{2}}{\left|1-\bar{\zeta} e^{i \theta}\right|^{2}}, \quad \zeta \in \mathbb{D} .
$$

Then for $0<r<1$ and $|\zeta|<r$, we have

$$
\frac{d}{d r}\left(r H\left(e^{i \theta}, \frac{\zeta}{r}\right)\right)>0 .
$$

Proof. For $0<r<1$ and $|\zeta|<r$ we have

$$
H\left(e^{i \theta}, \frac{\zeta}{r}\right)=\frac{\left(r^{2}-|\zeta|^{2}\right)^{2}}{r^{2} \mid r-\bar{\zeta} e^{\left.i \theta\right|^{2}}}
$$

A computation reveals that

$$
\frac{d}{d r} H\left(e^{i \theta}, \frac{\zeta}{r}\right)=4 \frac{r^{2}-|\zeta|^{2}}{r\left|r-\bar{\zeta} e^{i \theta}\right|^{2}}-\frac{\left(r^{2}-|\zeta|^{2}\right)^{2}}{\left|r-\bar{\zeta} e^{i \theta}\right|^{2}}\left(\frac{2}{r^{3}}+\frac{1}{r^{2}\left(r-\bar{\zeta} e^{i \theta}\right)}+\frac{1}{r^{2}\left(r-\zeta e^{-i \theta}\right)}\right),
$$

and therefore

$$
r \frac{d}{d r} H\left(e^{i \theta}, \frac{\zeta}{r}\right)=\frac{4\left(r^{2}-|\zeta|^{2}\right)}{\left|r-\bar{\zeta} e^{i \theta}\right|^{2}}-\frac{\left(r^{2}-|\zeta|^{2}\right)^{2}}{\left|r-\bar{\zeta} e^{i \theta}\right|^{2}}\left(\frac{2}{r^{2}}+\frac{1}{r\left(r-\bar{\zeta} e^{i \theta}\right)}+\frac{1}{r\left(r-\zeta e^{-i \theta}\right)}\right) .
$$

It then follows that

$$
\begin{aligned}
& \frac{d}{d r}\left(r H\left(e^{i \theta}, \frac{\zeta}{r}\right)\right)=H\left(e^{i \theta}, \frac{\zeta}{r}\right)+r \frac{d}{d r} H\left(e^{i \theta}, \frac{\zeta}{r}\right) \\
&=\frac{\left(r^{2}-|\zeta|^{2}\right)^{2}}{r\left|r-\bar{\zeta} e^{i \theta}\right|^{2}}\left(\frac{r-|\zeta|}{r(r+|\zeta|)}+\frac{2}{r-|\zeta|}-\frac{1}{r-\bar{\zeta} e^{i \theta}}-\frac{1}{r-\zeta e^{-i \theta}}\right) \\
& \quad \geq \frac{\left(r^{2}-|\zeta|^{2}\right)^{2}(r-|\zeta|)}{r^{2}\left|r-\bar{\zeta} e^{i \theta}\right|^{2}(r+|\zeta|)}>0,
\end{aligned}
$$

because for $|\zeta|<r$ we have

$$
\frac{1}{r-\zeta e^{-i \theta}}+\frac{1}{r-\bar{\zeta} e^{i \theta}}=2 \operatorname{Re}\left(\frac{1}{r-\zeta e^{-i \theta}}\right) \leq \frac{2}{\mid r-\zeta e^{-i \theta \mid}} \leq \frac{2}{r-|\zeta|} .
$$

The proof is now complete.

In the next lemma we prove that $r \Gamma\left(z, \frac{\zeta}{r}\right)$ has the same monotonicity property as well.

Lemma 3.2. For $0<r<1, z \in \mathbb{D}$, and $|\zeta|<r$, the function $r \Gamma\left(z, \frac{\zeta}{r}\right)$ is an increasing function of $r$. 
Proof. We shall use the following formula, due to Hadamard, which represents the biharmonic Green function $\Gamma(z, \zeta)$ in terms of the harmonic compensator $H(z, \zeta)$ (see [6], p. 74):

$$
\Gamma(z, \zeta)=\frac{1}{\pi} \int_{\max \{|z|,|\zeta|\}}^{1} \int_{-\pi}^{\pi} H\left(e^{i \theta}, \frac{z}{s}\right) H\left(e^{i \theta}, \frac{\zeta}{s}\right) s d \theta d s, \quad(z, \zeta) \in \mathbb{D} \times \mathbb{D} .
$$

Let us denote by

$$
K(z, \zeta)=\chi_{[\max \{|z|,|\zeta|\}, 1]}, \quad(z, \zeta) \in \mathbb{D} \times \mathbb{D},
$$

where $\chi_{I}$ stands for the characteristic function of the interval $I$. Assuming that $|\zeta|<r$, we obtain

$$
r \Gamma\left(z, \frac{\zeta}{r}\right)=\frac{1}{\pi} \int_{0}^{1} \int_{-\pi}^{\pi} K\left(z, \frac{\zeta}{r}\right) H\left(e^{i \theta}, \frac{z}{s}\right) r s H\left(e^{i \theta}, \frac{\zeta}{r s}\right) d \theta d s .
$$

It is easy to see that the function $K\left(z, \frac{\zeta}{r}\right)$ increases with $r$. On the other hand, since $\frac{|\zeta|}{r}<s$, we have $|\zeta|<r s$, and hence the function

$$
r s H\left(e^{i \theta}, \frac{|\zeta|}{r s}\right)
$$

increases with $r$, according to Lemma 3.1. This completes the proof.

Proposition 3.3. Let $w$ be a superbiharmonic weight function satisfying the condition $0 \leq w(z) \leq C(1-|z|)$. Then the function $r w\left(\frac{z}{r}\right)$, for $0<r<1$ and $|z|<r$, is increasing in $r$.

Proof. This is an immediate consequence of Theorem 2.6, Lemma 3.1, and Lemma 3.2 .

Lemma 3.4. Suppose that $\mu$ is a finite positive measure on a measure space $X, 0<$ $p<+\infty$, and $f_{n}, f$ are measurable functions such that

$$
\limsup _{n \rightarrow+\infty} \int_{X}\left|f_{n}\right|^{p} d \mu \leq \int_{X}|f|^{p} d \mu<+\infty,
$$

and $f_{n} \rightarrow f$ almost everywhere with respect to the measure $\mu$. Then

$$
\lim _{n \rightarrow+\infty} \int_{X}\left|f-f_{n}\right|^{p} d \mu=0 .
$$

Proof. This is a well-known statement; for a proof the reader is referred to [3], p. 21 , or [7], p. 66.

We can now state the main result of this section.

Theorem 3.5. $(0<p<+\infty)$ Let $w$ be a superbiharmonic weight function satisfying the condition $0 \leq w(z) \leq C(1-|z|)$. Then the polynomials are dense in the weighted Bergman space $L_{a}^{p}(\mathbb{D}, w)$.

Proof. Let $f$ be a function in the weighted Bergman space $L_{a}^{p}(\mathbb{D}, w), 0<p<+\infty$. We intend to prove that the function $f$ can be approximated by the polynomials in norm. Since the dilation $f_{r}$ for every $0<r<1$ can be approximated in norm by the polynomials, it suffices to show that $f_{r} \rightarrow f$ in norm as $r \rightarrow 1^{-}$. It is easy to see that $f_{r} \rightarrow f$ pointwise, hence what we need to verify is

$$
\left\|f_{r}\right\|_{L_{a}^{p}(\mathbb{D}, w)} \rightarrow\|f\|_{L_{a}^{p}(\mathbb{D}, w)}, \quad \text { as } \quad r \rightarrow 1^{-} .
$$


This together with the standard Lemma 3.4 implies that

$$
\left\|f_{r}-f\right\|_{L_{a}^{p}(\mathbb{D}, w)} \rightarrow 0, \quad \text { as } \quad r \rightarrow 1^{-},
$$

from which the result follows. We start by writing

$$
\left\|f_{r}\right\|_{L_{a}^{p}(\mathbb{D}, w)}^{p}=\int_{\mathbb{D}}\left|f_{r}(z)\right|^{p} w(z) d A(z) .
$$

We now replace $z$ with $z / r$ on the right side of the above formula to obtain

$$
\left\|f_{r}\right\|_{L_{a}^{p}(\mathbb{D}, w)}^{p}=\frac{1}{r^{3}} \int_{r \mathbb{D}}|f(z)|^{p} r w\left(\frac{z}{r}\right) d A(z) .
$$

According to Proposition 3.3 the integrand is an increasing function of $r$, hence we can apply the monotone convergence theorem to conclude that

$$
\left\|f_{r}\right\|_{L_{a}^{p}(\mathbb{D}, w)}^{p} \rightarrow\|f\|_{L_{a}^{p}(\mathbb{D}, w)}^{p}, \quad \text { as } \quad r \rightarrow 1^{-},
$$

from which the theorem follows.

\section{ACKNOWLEDGEMENT}

I wish to thank Professor H. Hedenmalm of Lund University, Sweden, for the discussions we had.

\section{REFERENCES}

1. A. Abkar, H. Hedenmalm, A Riesz representation formula for super-biharmonic functions, Ann. Acad. Sci. Fenn. Math. 26 (2001), 305-324. MR 2002c:31001

2. A. Aleman, S. Richter and C. Sundberg, Beurling's theorem for the Bergman space, Acta Math. 177 (1996), 275-310. MR 98a:46034

3. P. L. Duren, Theory of $H^{p}$ spaces, Academic Press, New York, 1970. MR 42:3552

4. P. R. Garabedian, Partial differential equations, John Wiley and Sons, New York, London, and Sydney, 1964. MR 28:5247

5. V. P. Havin and N. K. Nikolski, Linear and complex analysis. Problem book 3. Part II, Lecture Notes in Mathematics, 1574, Springer-Verlag, Berlin, 1994. MR 96c:00001b

6. H. Hedenmalm, A computation of Green functions for the weighted biharmonic operators $\Delta|z|^{-2 \alpha} \Delta$, with $\alpha>-1$, Duke Math. J. 75 (1994), 51-78. MR 95k:31005

7. H. Hedenmalm, B. Korenblum, K. Zhu, Theory of Bergman spaces, Graduate Texts in Mathematics, 199, Springer-Verlag, New York, 2000. MR 2001c:46043

8. K. Hoffman, Banach spaces of analytic functions, Dover Publications, Inc., New York, 1988. MR 92d:46066

9. S. N. Mergelyan, On completeness of systems of analytic functions, Amer. Math. Soc. Translations 19 (1962), 109-166.

10. W. Rudin, Real and complex analysis, third edition, McGraw-Hill Book Company, Singapore, 1987. MR 88k:00002

Department of Mathematics, Imam Khomeini International University, P.O. Box 288, QAZVIN 34194, IRAN

Current address: Department of Mathematics, Institute for Studies in Theoretical Physics and Mathematics, P.O. Box 19395-1795, Tehran, Iran

E-mail address: abkar@ipm.ir 